\title{
Prevalence and factors associated with extended-spectrum $\beta$ - lactamase producing Enterobacteriaceae bacteraemia in University Hospital of Befelatanana, Madagascar
}

\author{
${ }^{* 1}$ Rakotovao-Ravahatra Z. D., ${ }^{2}$ Randriatsarafara, F. M., ${ }^{3}$ Rakotovao, A. L., and \\ ${ }^{3}$ Rasamindrakotroka, A.

\begin{abstract}
${ }^{1}$ Laboratory of Joseph Raseta Befelatanana, University Hospital, Antananarivo, Madagascar ${ }^{2}$ Public Health Department, Faculty of Medicine, University of Antananarivo, Madagascar
\end{abstract} \\ ${ }^{3}$ Medical Biology Department, Faculty of Medicine, University of Antananarivo, Madagascar \\ *Correspondence to: ravahatradomoina@yahoo.fr; +261340930120
}

\begin{abstract}
:
Background: The extended-spectrum $\beta$-lactamase (ESBL) producing Enterobacteriaceae are a major cause of nosocomial bacteraemia. The objectives of this study are to describe the antibiotic resistance pattern of ESBL producing Enterobacteriaceae responsible for bacteraemia and identify factors associated with these infections in a University Hospital in Madagascar.

Methodology: This is a descriptive cross-sectional study of 300 randomly selected patients with clinical features of bacteraemia whose blood cultures were processed for isolation and identification of bacterial pathogens over a period of six months (October 2019 to March 2020) at the laboratory of the University Hospital of Befelatanana. Blood culture samples were processed by conventional microbiological method for isolation of Enterobacteriaceae, which were identified to species level using Analytical Profile Index (API) $20 E \AA$ test system. Antibiotic susceptibility of each isolate was performed by the disk diffusion technique and ESBL production was detected by the 'synergy' method.

Results: Of the 300 patients, 54 were positive for bacteria, giving a prevalence rate of $18 \%$ for microbiologically documented bacteraemia. Of the 54 bacterial pathogens, Enterobacteriaceae isolates constituted $37(68.5 \%)$, with $23(42.6 \%)$ being ESBL producing and $14(25.9 \%)$ non-ESBL producing isolates, $14(25.9 \%)$ were staphylococci and $3(5.6 \%)$ were streptococci isolates. All $23(100 \%)$ ESBL producing Enterobacteriaceae isolates were resistant to amoxicillin, amoxicillin-clavulanic acid and the third generation cephalosporins (3GC), $19(82.6 \%)$ to gentamycin and $18(78.3 \%)$ to cotrimoxazole. On the other hand, the non-ESBL producing isolates were more sensitive because only $10(71 \%)$ were resistant to amoxicillin, $7(50 \%)$ to cotrimoxazole, $2(14 \%)$ to amoxicillin-clavulanic acid, $1(7.1 \%)$ to gentamycin, and none $(0 \%)$ was resistant to 3GC. All 54 Enterobacteriaceae isolates were sensitive to amikacin and imipenem. Age less than 20 years $(93.8 \%)(p=0.001)$ and hospitalization in intensive care units $(90.9 \%)(p=0.04)$ were significant risk factors associated with infection by ESBL producing Enterobacteriaceae.

Conclusion: ESBL producing Enterobacteriaceae responsible for bacteraemia in University Hospital of Befelatanana, Madagascar, are resistant to many classes of antibiotics. Carbapenems and amikacin are the antibiotics of choice.
\end{abstract}

Keywords: ESBL, Enterobacteriaceae, bacteraemia, antibiotic resistance

Received April 28, 2020; Revised August 9, 2020; Accepted August 19, 2020

Copyright 2021 AJCEM Open Access. This article is licensed and distributed under the terms of the Creative Commons Attrition 4.0 International License <a rel="license" href="http://creativecommons.org/licenses/by/4.0/", which permits unrestricted use, distribution and reproduction in any medium, provided credit is given to the original author(s) and the source. Editor-in-Chief: Prof. S. S. Taiwo

\section{Prévalence et facteurs associés à la bactériémie à entérobactéries productrices de $\beta$-lactamases à spectre étendu dans l'hôpital universitaire de Befelatanana, Madagascar}

\author{
${ }^{* 1}$ Rakotovao-Ravahatra Z. D., ${ }^{2}$ Randriatsarafara, F. M., ${ }^{3}$ Rakotovao, A. L., et \\ ${ }^{3}$ Rasamindrakotroka, A.
}

\footnotetext{
${ }^{1}$ Laboratoire Joseph Raseta Befelatanana, Hôpital Universitaire, Antananarivo, Madagascar 2Département de Santé Publique, Faculté de Médecine, Université d'Antananarivo, Madagascar
} 
${ }^{3}$ Département de Biologie Médicale, Faculté de Médecine, Université d'Antananarivo, Madagascar *Correspondance à: ravahatradomoina@yahoo.fr; +2613409301 20

\begin{abstract}
Abstrait:
Contexte: Les entérobactéries productrices de $\beta$-lactamases à spectre étendu (BLSE) sont une cause majeure de bactériémie nosocomiale. Les objectifs de cette étude sont de décrire le profil de résistance aux antibiotiques des entérobactéries productrices de BLSE responsables de bactériémie et d'identifier les facteurs associés à ces infections dans un hôpital universitaire de Madagascar.

Méthodologie: Il s'agit d'une étude transversale descriptive de 300 patients sélectionnés au hasard présentant des caractéristiques cliniques de bactériémie dont les hémocultures ont été traitées pour l'isolement et l'identification des bactéries pathogènes sur une période de six mois (octobre 2019 à mars 2020) au laboratoire du Hôpital universitaire de Befelatanana. Les échantillons d'hémoculture ont été traités par une méthode microbiologique conventionnelle pour l'isolement des entérobactéries, qui ont été identifiées au niveau de l'espèce à l'aide du système de test Analytical Profile Index (API) 20E $囚$. La sensibilité aux antibiotiques de chaque isolat a été réalisée par la technique de diffusion sur disque et la production de BLSE a été détectée par la méthode «synergie».

Résultats: Sur les 300 patients, 54 étaient positifs pour les bactéries, ce qui donne un taux de prévalence de $18 \%$ pour une bactériémie microbiologiquement documentée. Parmi les 54 bactéries pathogènes, les isolats d'entérobactéries constituaient $37(68,5 \%), 23(42,6 \%)$ produisant des BLSE et $14(25,9 \%)$ isolats ne produisant pas de BLSE, 14 (25,9\%) étaient des staphylocoques et $3(5,6 \%)$ l'étaient isolats de streptocoques. Les 23 isolats $(100 \%)$ de BLSE produisant des Enterobacteriaceae étaient tous résistants à l'amoxicilline, à l'amoxicilline-acide clavulanique et aux céphalosporines de troisième génération (3GC), $19(82,6 \%)$ à la gentamycine et $18(78,3 \%)$ au cotrimoxazole. En revanche, les isolats non producteurs de BLSE étaient plus sensibles car seuls $10(71 \%)$ étaient résistants à l'amoxicilline, $7(50 \%)$ au cotrimoxazole, $2(14 \%)$ à l'amoxicilline-acide clavulanique, $1(7,1 \%)$ à la gentamycine, et aucun $(0 \%)$ n'était résistant à la $3 G C$. Les 54 isolats d'Enterobacteriaceae étaient tous sensibles à l'amikacine et à l'imipénème. L'âge de moins de 20 ans $(93,8 \%)(p=0,001)$ et l'hospitalisation en unité de soins intensifs $(90,9 \%)(p=0,04)$ étaient des facteurs de risque importants associés à l'infection par les entérobactéries productrices de BLSE.

Conclusion: Les entérobactéries productrices de BLSE responsables de bactériémie à l'hôpital universitaire de Befelatanana, Madagascar, sont résistantes à de nombreuses classes d'antibiotiques. Les carbapénèmes et l'amikacine sont les antibiotiques de choix.
\end{abstract}

Mots clés: BLSE, entérobactéries, bactériémie, résistance aux antibiotiques

\section{Introduction:}

Bacteraemia is defined as the presence of bacteria in the blood. The blood is normally sterile environment, therefore the detection of bacteria in the blood is always abnormal. The annual incidence of community-onset bacteraemia is reported to be between 40 and 154 per 100,000 populations (1). Bacteraemia is classified as nosocomial or community acquired, in accordance with the classic CDC criteria (2). Among bacterial isolates responsible for bacteraemia, the extended spectrum $\beta$-lactamase (ESBL) producing Enterobacteriaceae are highly implicated. Indeed, community and hospital-acquired ESBL producing Enterobacteriaceae are prevalent worldwide (3). Reliable identification of ESBL producing organisms in clinical laboratories can be challenging, therefore their prevalence is likely to be underestimated (3). ESBL are enzymes that confer resistance to most $\beta$ lactam antibiotics, including penicillins, cephalosporins, and monobactam (aztreonam). Infections caused by ESBL producing bacterial pathogens have been associated with poor clinical outcomes (3).

Bacteraemia have several important health consequences. The immune response to the bacteria can cause sepsis and septic shock especially in Gram negative bacteria, which is associated with high mortality (4).
Bacteria can also spread via the blood to other parts of the body, causing infections far away from the original site of infection, such as endocarditis and osteomyelitis (5). Antimicrobial therapy of bacteraemia caused by ESBL-producing organisms presents additional challenge, because these organisms are also often resistant to other anti microbials such as trimethoprim-sulfamethoxazole, aminoglycosides and the fluoroquinolones.

Until recently, the major problems posed by ESBL-producing organisms were related to nosocomial infections caused by Klebsiella pneumoniae, which produced mainly the TEM and SHV types of ESBL (6). Similarly, ESBL production is one of the most important resistance mechanisms that hinder antimicrobial therapy of infections caused by the Enterobacteriaceae (7). Therefore, it is imperative to quantify the problem, and reinforce guidelines promoting appropriate antibiotic use. Antibiotic resistance studies of these ESBL-producing Enterobacteriaceae should hence be regularly conducted to improve patient management. The objectives of this study are to describe the antibiotic resistance profile of ESBL-producing Enterobacteriaceae responsible for bacteraemia and to identify risk factors associated with these infections in University Hospital of Befelatanana, Madagascar. 


\section{Materials and method:}

\section{Study setting}

This study was carried out at the laboratory of the University Hospital of Befelatanana, located in Antananarivo, the capital city of Madagascar. This laboratory is versatile and performs haematological, immunological, biochemical and microbiological analyses on clinical samples.

\section{Study design, subjects and sample size}

This study was a descriptive crosssectional design involving 300 patients with clinical suspicion of blood stream infections (BSI) randomly recruited for the study over a period of six months (October 2019 to March 2020), and whose blood culture samples were processed in the laboratory of the University Hospital of Befelatanana. BSI was confirmed in subjects who had clinical features compatible with systemic inflammatory response syndrome, and isolation of bacteria in at least one blood culture bottle (8).

\section{Blood specimen collection}

Blood samples were collected from the antecubital fossa of each patient at the peak of fever by a trained nurse under strict aseptic condition using sterile needles and syringes. Three blood samples per patient (paired samples of aerobic and anaerobic) were collected within 24 hours, and between 5 to $10 \mathrm{ml}$ of blood was collected for each sample. The samples were inoculated into the blood culture bottles (Oxoid B0100Mß, UK) using new set of sterile needles with thorough mixing of the content, for aerobic, anaerobic and micro-aerophilic incubation. The inoculated bottles were transported immediately to the laboratory.

\section{Processing of blood culture for isolation of Enterobacteriaceae}

Blood culture was performed with the conventional culture technique. All inoculated culture bottles were incubated at $37^{\circ} \mathrm{C}$ in aerobic, anaerobic and micro-aerophilic environment. Culture bottles were examined daily by laboratory technicians for evidence of bacterial growth, which is shown by positive pressure growth indicator device connected to the culture bottles. A positive pressure in the bottle displaces a quantity of blood/broth mixture into the chamber as a sign of microbial activity, and this was indicated when the blood/broth mixture rises above the green locking sleeve of the growth indicator device. Blood culture result was adjudged negative if there was no evidence of growth after 5 days of incubation

\section{Bacteria identification from positive blood cultures \\ Positive blood cultures were sub-} cultured on blood, chocolate and chromogenic agar (Uriselect $($ ) ) to obtain discrete colonies. The Uriselect $\AA$ agar was used to preliminarily identify Enterobacteriaceae through specific staining of bacterial colonies on this medium. The confirmatory biochemical identification of the Enterobacteriaceae was done using the

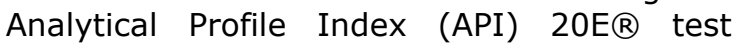
system.

\section{Antibiotic susceptibility testing}

Antibiotic susceptibility testing (AST) was performed on Mueller-Hinton agar using the disk diffusion method in accordance with the recommendations of the "Comité de l'antibiogramme de la Société Française de Microbiologie" (9). The MH agar plate was inoculated with 0.5 McFarland standards suspension of each Enterobacteriaceae isolate, and antibiotic disks were placed on the plate and incubated aerobically at $37^{\circ} \mathrm{C}$ for 24 hours. The diameter of zone of inhibition to each disk was read using a calibrated ruler and the result interpreted as sensitive or resistant according to the guidelines of the "Comité de l'antibiogramme de la Société Française de Microbiologie" (9).

The antibiotic disks used included amoxicillin $(20 \mu \mathrm{g})$, amoxicillin-clavulanic acid $(20 \mu \mathrm{g} / 10 \mu \mathrm{g})$, 3rd generation cephalosporins (cefixime $5 \mu \mathrm{g}$, ceftazidime $30 \mu \mathrm{g}$, ceftriaxone $30 \mu \mathrm{g}$, and cefotaxime $30 \mu \mathrm{g})$, imipenem $(10 \mu \mathrm{g})$, aminoglycosides (gentamycin $10 \mu \mathrm{g}$, tobramycin $10 \mu \mathrm{g}$, and amikacin $30 \mu \mathrm{g})$, quinolones (nalidixic acid $30 \mu \mathrm{g}$, ciprofloxacin $5 \mu \mathrm{g}$, and levofloxacin 5 $5 \mathrm{~g}$ ), trimethoprimsulfamethoxazole $(1.25 \mu \mathrm{g} / 23.75 \mu \mathrm{g})$ and chloramphenicol $(30 \mu \mathrm{g})$.

\section{Detection of ESBL production}

ESBL production was detected by the 'synergy' method using amoxicillin/clavulanic acid disk $(20 / 10 \mu \mathrm{g})$ placed at $30 \mathrm{~mm}$ center to center of ceftazidime disk $(30 \mu \mathrm{g})$, cefotaxime disk $(30 \mu \mathrm{g})$, ceftriaxone disk $(30 \mu \mathrm{g})$ and aztreonam disk (30 $\mathrm{gg})$ on $\mathrm{MH}$ agar plate that has been inoculated with 0.5 McFarland standards suspension of the Enterobacteriaceae isolates. After 24 hours aerobic incubation at $37^{\circ} \mathrm{C}$, a "champagne cork" image indicates ESBL production (10). Klebsiella pneumoniae ATCC 700603 was used as control strain (11).

\section{Data collection, entry and statistical analysis}

The study parameters of each subject obtained were gender, age, unit/department, bacteriological results of blood culture and the results of antibiogram, which were obtained 
from the laboratory case files. Each patient had previously completed analysis request form which contains all the required demographic and clinical information, which were transcribed into the laboratory case files. Data entry and processing were performed on EPI-INFO 3.5.2 statistical software. The comparison of variables was done using the Chi square test, and statistical significance threshold used was $p<0.05$.

\section{Ethical approval}

The study was approved by the Director of Establishment of the University Hospital of Befelatanana and the Department Head of the Laboratory. This study respected the notion of anonymity and confidentiality.

\section{Results :}

Of the total of 300 patients with clinical evidence of blood stream infections recruited in this study, blood culture sample was positive for bacteria in 54 patients, giving a prevalence rate of $18 \%$ for bacteraemia. Of the 54 bacterial isolates, 37 $(68.5 \%)$ were Enterobacteriaceae with 23 (42.6\%) ESBL producing and 14 (25.9\%) non-ESBL producing, 14 (25.9\%) were staphylococci and $3(5.6 \%)$ were streptococci isolates (Fig 1).

The 23 ESBL producing Enterobacteriaceae were represented by 11 (47.8\%) Klebsiella pneumoniae, 8 (34.8\%) Enterobacter cloacae and 4 (17.4\%) Escherichia coli isolates (Fig 2). The 14 non-ESBL producing isolates were represented by $6(42 \%)$ E. coli, $3(21.4 \%)$ E. cloacae, $2(14.3 \%)$ K. pneumoniae, 1 (7.1\%) Serratia marcescens, $1(7.1 \%)$ Salmonella Typhi and 1 (7.1\%) Shigella dysenteriae isolates (Fig 3).

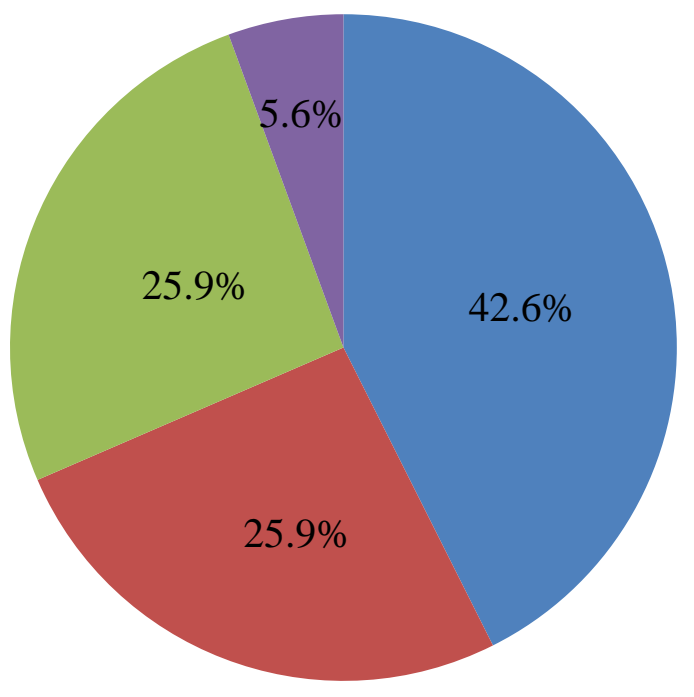

- ESBL-producing Enterobacteriaceae

non-ESBL producing Enterobacteriaceae - Staphylococci

- Streptococci

Fig 1: Bacteriological results of the blood culture among patients with bacteraemia in University Hospital, Befelatanana, Madagascar 


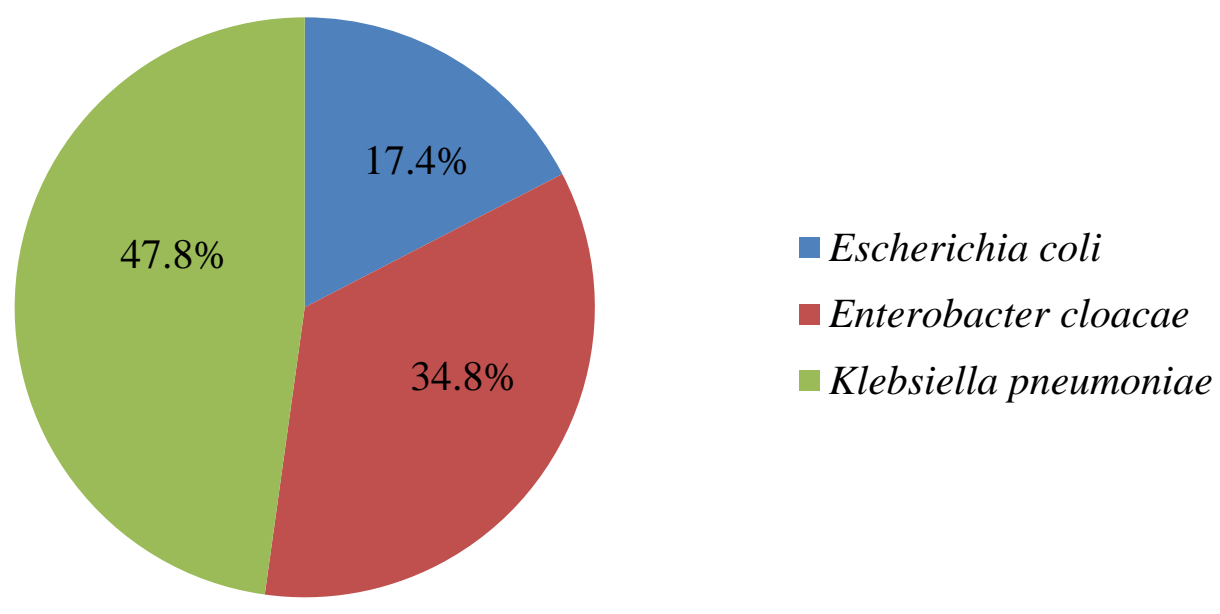

Fig 2: Species of ESBL producing Enterobacteriaceae causing bacteraemia in University Hospital, Befelatanana, Madagascar

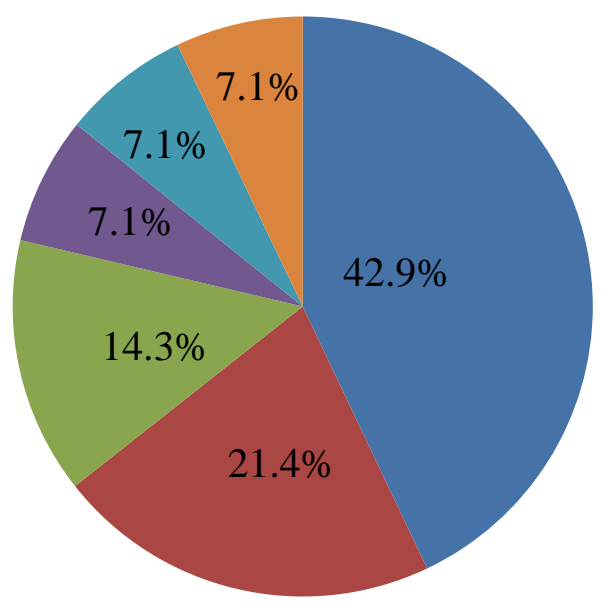

- Escherichia coli

- Enterobacter cloacae

- Klebsiella pneumoniae

- Salmonella typhi

- Serratia marcescens

- Shigella dysenteriae

Fig 3: Species of non-ESBL producing Enterobacteriaceae isolates causing bacteraemia in University Hospital, Befelatanana, Madagascar

Regarding antibiotic resistance of the Enterobacteriaceae, resistance rate varies from $0 \%$ to $100 \%$ for all of the antibiotics tested. All 23 (100\%) ESBL producing Enterobacteriaceae were resistant to amoxicillin, amoxicillin-clavulanic acid, and third generation cephalosporins, $19(82.6 \%)$ were resistant to gentamycin and $18(78.3 \%)$ to cotrimoxazole. On the other hand, the non-ESBL producing Enterobacteriaceae were more sensitive because only $10(71 \%)$ were resistant to amoxicillin, 7 (50\%) to cotrimoxazole, 2
(14\%) to amoxicillin-clavulanic acid, 1 (7.1\%) to gentamycin and no isolate was resistant to third generation cephalosporins.

All Enterobacteriaceae isolates were sensitive to amikacin and imipenem (Fig 4). Age below 20 years (15 of 16 patients, 93 . $8 \%)(p=0.001)$, and hospitalization in intensive care units (10 of 11 patients, $90.9 \%$ ) $(p=0.04)$ were significantly associated with ESBL producing Enterobacteriaceae bacteraemia (Table 1). 


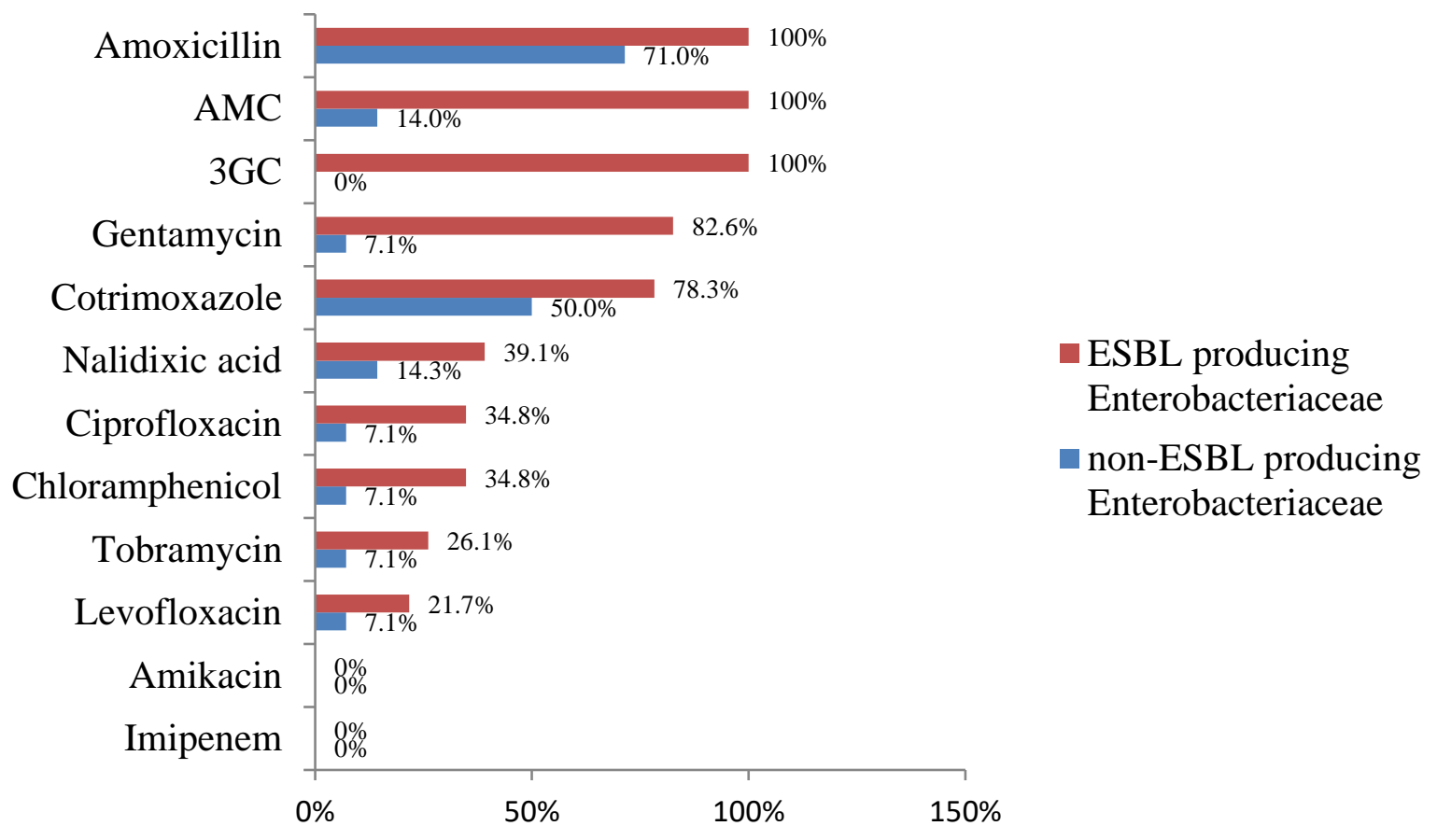

Fig 4: Antibiotic resistance of ESBL and non-ESBL producing Enterobacteriaceae causing bacteraemia in University Hospital, Befelatanana, Madagascar

Table 1: Factors associated with ESBL-producing Enterobacteriaceae bacteraemia in University Hospital, Befelatanana, Madagascar

\begin{tabular}{|c|c|c|c|c|c|c|}
\hline & \multicolumn{2}{|c|}{$\begin{array}{c}\text { ESBL-producing } \\
\text { Enterobacteriaceae } \\
(n=23)\end{array}$} & \multicolumn{2}{|c|}{$\begin{array}{c}\text { Non-ESBL producing } \\
\text { Enterobacteriaceae }(n=14)\end{array}$} & \multirow[t]{2}{*}{ Total $(n=37)$} & \multirow[t]{2}{*}{$p$ value } \\
\hline \multicolumn{5}{|l|}{ Age (years) } & & \\
\hline$>20$ & 8 & 38.1 & 13 & 61.9 & 21 & $0.001^{s}$ \\
\hline$\leq 20$ & 15 & 93.8 & 1 & 6.3 & 16 & \\
\hline \multicolumn{7}{|l|}{ Gender } \\
\hline Female & 15 & 62.5 & 9 & 37.5 & 24 & $0.76^{\mathrm{ns}}$ \\
\hline Male & 8 & 61.5 & 5 & 38.5 & 13 & \\
\hline \multicolumn{7}{|l|}{ Department } \\
\hline Intensive care unit & 10 & 90.9 & 1 & 9.1 & 11 & $0.04^{\mathrm{s}}$ \\
\hline Internal medicine & 13 & 50.0 & 13 & 50.0 & 26 & \\
\hline
\end{tabular}

\section{Discussion:}

This study reports a prevalence rate of $18 \%$ for microbiologically documented bacteraemia in University Hospital of Befelatanana, Madagascar, which included patients of all age groups in different departments/units of the hospital. The rate in our study is higher than the $3.3 \%$ reported by Jamil et al., (12) among patients with chronic kidney disease, and $2.9 \%$ reported by Biondi et al., (13) among febrile neonates. However, the prevalence rate in our study is lower compared to the study conducted by Adeyemo et al., (14) who reported bacteraemia prevalence rates of $40.9 \%, 33.3 \%$, and 
$50 \%$ in cleft lip surgery, cleft palate surgery, and alveoloplasty respectively. Indeed, bacteria can enter the blood stream as a severe complication of infections such as pneumonia or meningitis, during surgery (especially involving mucous membranes such as gastrointestinal tract), or due to catheters and other foreign bodies inserted into the blood vessels, including during intravenous injection drug abuse (15). Transient bacteraemia can also result after dental procedures or brushing of teeth (16).

Our study reported $42.6 \%$ (23 of 54 ) Enterobacteriaceae isolates causing bacteraemia to be ESBL-producing. This is lower than $73.3 \%$ rate reported among 131 bacteraemic patients in a study carried out in South India (7). Indeed, we found many other bacteria responsible for bacteraemia in this study such as streptococci and staphylococci. ESBLs are plasmid encoded $\beta$-lactamases that confer resistance to penicillins, cephalosporins, and aztreonam, which are frequently found in Klebsiella species, E. coli, and other enterobacteria (6). Our study reported high frequency of ESBL-producing Enterobacteriaceae among species of $K$. pneumoniae, $E$, coli and $E$. cloacae but some species were nonESBL producing isolates and these included S. marcescens, S. Typhi and S. dysenteriae. The antibiotic susceptibility (AST) profile indicated that the ESBL producing Enterobacteriaceae isolates were more resistant than the non-ESBL producing isolates. In addition to their resistance to majority of the $\beta$-lactam agents, the ESBL producing isolates were also more resistant to other classes of antibiotics such as cotrimoxazole, aminoglycosides (gentamycin, tobramycin), quinolones (nalidixic acid, ciprofloxacin, levofloxacin) and chloramphenicol, than the nonESBL producing isolates. Indeed, ESBL producing Enterobacteriaceae are frequently found in hospital and are responsible for several nosocomial infections (17). These antibiotic resistant bacteria had emerged under the pressure of frequent use and misuse of certain antibiotics in the hospitals. Cefotaxime is one of the most commonly used oxyimino- $\beta$-lactam, a fact that may have favored the selection of CTX-M ESBL producing isolates, because such types of ESBL are mainly cefotaximases $(6,18)$.

The fluoroquinolone use and misuse, which is also frequent, has been identified as a risk factor for other infections caused by ESBL-producing E. coli $(19,20)$, probably as a result of co-selection (21). The empiric use of the 3rd and 4th generation cephalosporins should be curtailed, as cephalosporin use was associated with an increased risk of ESBL production. A study conducted by Hsieh et al., (22) showed that significant risk factors associated with bacteraemia by ESBL produ- cing E. coli included recent antibiotic exposure (within 30 days) and urinary catheter placement. In view of their excellent in vitro activity, carbapenems should be the initial empiric choice for serious life-threatening infections caused by Enterobacteriaceae, with prompt de-escalation when culture and susceptibility results become available (7). Indeed, no Enterobacteriaceae isolate was resistant to carbapenems (imipenem) in our study, and similarly, all isolates were susceptible to amikacin. A study in Douala also found that imipenem (with $1.3 \%$ resistance) and amikacin (with $12.9 \%$ resistance rate) were the most effective antibiotics against enterobacteria (23). Taking together, these data suggest that the penems (carbapenem, imipenem, meropenem) and aminoglycosides especially amikacin could be an effective alternative choice for the treatment of ESBLproducing Enterobacteriaceae bacteraemia. With regards to risk factors for Enterobacteriaceae bacteraemia, subjects under 20 years of age and those hospitalized in the intensive care units were most frequently infected by ESBL-producing isolates $(p<0.05)$. Studies have shown that children are more fragile and more likely to be infected by ESBL producing Enterobacteriaceae (24). Similarly, invasive procedures such as mechanical ventilation, tracheostomy or the use of catheters favor nosocomial infections including bacteraemia in the intensive care units (25). Thus, infection prevention and control measures must be reinforced in intensive care units to limit the spread of these resistant bacteria.

\section{Conclusion:}

ESBL producing Enterobacteriaceae are frequently responsible for nosocomial bacteraemia in Madagascar. These bacteria are resistant to many classes of antibiotics, while carbapenems and amikacin appear effective, and are the antibiotics of choice. Infection prevention and control measures must be reinforced in intensive care units to limit further emergence and spread of resistant bacteria.

\section{Acknowledgements:}

The authors appreciate with thanks all the staff of the laboratory of University Hospital of Befelatanana and all the laboratory technicians. The authors are equally grateful to the Director of Establishment for authorizing the conduct of this study.

\section{References:}

1. Viscoli, C. Blood stream infections: the peak of the iceberg. Virulence. 2016; 7: 248 - 251 
CDC definitions for nosocomial infections. Am J Infect Control. 1998; 16: 128140

3. Ben-Ami, R., Rodríguez-Baño, J., Arslan, H., et al. A multinational survey of risk factors for infection with extended-spectrum betalactamase-producing Enterobacteriaceae in nonhospitalized patients. Clin Infect Dis. 2009; 49: 682

4. Singer, M., Deutschman, C. S., Seymour, C. W., et al. The Third International Consensus Definitions for Sepsis and Septic Shock (Sepsis3). JAMA. 2016; 315: 801-810

5. Zeman, F., Koller, M., Schecklmann, M., et al. Tinnitus assessment by means of standardized self-report questionnaires: Psychometric properties of the Tinnitus Questionnaire (TQ), the Tinnitus Handicap Inventory (THI), and their short versions in an international and multi-lingual sample. Hith Qual Life Out. 2012; 10:128

6. Paterson, D. L., and Bonomo, R. A. Extendedspectrum beta-lactamases: a clinical update. Clin Microbiol Rev. 2005; 18: 657-686

7. Abhilash, K. P., Veeraraghavan, B., and Abraham O. C. Epidemiology and outcome of bacteraemia caused by extended spectrum betalactamase (ESBL)-producing Escherichia coli and Klebsiella spp in a tertiary care teaching hospital in south India. J Assoc Physicians India. 2010; 58: 13-17

8. American College of Chest Physicians/Society of Critical Care Medicine Consensus Conference. Definitions for sepsis and organ failure and guidelines for the use of innovative therapies in sepsis. Crit Care Med. 1992; 20: 864-874

9. Société Française de Microbiologie. Tables of critical concentrations for the interpretation of MICs and critical diameters of inhibition zones. In: CASFM/EUCAST: Société Française de Microbiologie Ed; 2019: 38-44

10. Sbiti, M., Lahmadi, K., and Louzi, L. Epidemiological profile of uropathogenic enterobacteria producing extended spectrum betalactamases. Pan Afr Med J. 2017; 28: 29

11. Simione, F. American Type Culture Collection: A Model for Biological Materials Resource Management. In: Uhlir, P. F. (ed). Designing the Microbial Research Commons. Proceedings of an International Symposium. National Research Council of the National Academies. Washington: The National Academies Press; 2011: 228

12. Jamil, B., Bokhari, M. T., Saeed, A., et al. Bacteraemia: Prevalence and antimicrobial resistance profiling in chronic kidney diseases and renal transplant patients. J Pak Med Assoc. 2016; 66: 705-709

13. Biondi, E. A., Lee, B., Ralston, S. L., et al. Prevalence of Bacteraemia and Bacterial Meningitis in Febrile Neonates and Infants in the Second Month of Life: A Systematic Review and Meta-analysis. JAMA Network Open. 2019; 2: e190874

14. Adeyemo, W. L., Adeyemi, M. O., Ogunsola, F. T., et al. Prevalence and bacteriology of bacteraemia associated with cleft lip and palate surgery. J Craniofac Surg. 2013; 24: 1126-1131

15. Sligl, W., Taylor, G., and Brindley, P. G. Five years of nosocomial Gram-negative bacteraemia in a general intensive care unit: epidemiology, antimicrobial susceptibility patterns, and outcomes. Int J Infect Dis. 2006; 10: 320-325

16. Perez-Chaparro, P. J., Meuric, V., De Mello, G., et al. Bacteraemia of oral origin. Rev Stom Chir Max Fac. 2011; 112: 300-303

17. Tian, L., Sun, Z., and Zhang, Z. Antimicrobial resistance of pathogens causing nosocomial blood stream infection in Hubei Province, China, from 2014 to 2016: a multi center retrospective study. BMC Publ HIth. 2018; 18
(1): 1121

18. Bonnet, R. Growing group of extended-spectrum beta-lactamase: the CTX-M enzymes. Antimicrob Agents Chemother. 2004; 48: 1-14

19. Rodrı' guez-Ban $\sim$ O, J., Navarro, M. D., Romero, L., et al. Epidemiology and clinical features of infections caused by extended-spectrum betalactamase-producing Escherichia coli in nonhospitalized patients. J Clin Microbiol. 2004; 42: 1089-1094

20. Rodri' guez-Ban ${ }^{2}$, J., Navarro, M. D., Romero, L., et al. Clinical and molecular epidemiology of extended-spectrum beta-lactamase producing Escherichia coli as a cause of nosocomial infection or colonization: implications for control. Clin Infect Dis. 2006; 42: 37-45

21. Pitout, J. D., Nordmann, P., Laupland, K. B., et al. Emergence of Enterobacteriaceae producing extended-spectrum beta-lactamases (ESBLs) in the community. J Antimicrob Chemother. 2005; 56: 52-59

22. Hsieh, C. J., Shen, Y. H., and Hwang, K. P. Clinical implications, risk factors and mortality following community-onset bacteraemia caused by extended-spectrum $\beta$-lactamase (ESBL) and non-ESBL producing Escherichia coli. J Microbiol Immunol Infect. 2010; 43: 240-248

23. Ebongue, C. O., Tsiazok, M. D., Nda Mefo'o, J. P., et al. Evolution of antibiotic resistance of Enterobacteriaceae isolated at the Douala General Hospital from 2005 to 2012. Pan Afr Med J. 2015; 20: 227

24. Kaarme, J., Riedel, H., Schaal, W., et al. Rapid Increase in Carriage Rates of Enterobacteriaceae Producing Extended-Spectrum $\beta$-Lactamases in Healthy Preschool Children, Sweden. Emerg Infect Dis. 2018; 24: 1874-1881

25. Merzougui, L., Barhoumi, T., Guizani, T., et al. Nosocomial infections in the Intensive Care Unit: annual incidence rate and clinical aspects. Pan Afr Med. J. 2018; 30: 143 